\title{
Spread Pattern Formation of H5N1-Avian Influenza and its Implications for Control Strategies
}

\author{
R. Liu ${ }^{a}$, V. R. S. K. Duvvuri ${ }^{b}$, and J. Wu $\mathbf{u}^{b, c 1}$ \\ ${ }^{a}$ Department of Mathematics, Purdue University, West Lafayette, IN 47907, USA \\ ${ }^{b}$ Center for Disease Modeling, York Institute for Health Research \\ ${ }^{c}$ Department of Mathematics and Statistics, York University, Toronto, M3J 1L3, ON, Canada
}

\begin{abstract}
Mechanisms contributing to the spread of avian influenza seem to be well identified, but how their interplay led to the current worldwide spread pattern of H5N1 influenza is still unknown due to the lack of effective global surveillance and relevant data. Here we develop some deterministic models based on the transmission cycle and modes of H5N1 and focusing on the interaction among poultry, wild birds and environment. Some of the model parameters are obtained from existing literatures, and others are allowed to vary in order to assess the effectiveness of various control strategies involving bird migration, agro-ecological environments, live and dead poultry trading, smuggling of wild birds, mechanical movement of infected materials and specific farming practices. Our simulations are carried out for a set of parameters that leads to the basic reproduction number 3.3. We show that by reducing $95 \%$ of the initial susceptible poultry population or by killing all infected poultry birds within one day, one may control the disease outbreak in a local setting. Our simulation shows that cleaning the environment is also a feasible and useful control measure, but culling wild birds and destroying their habitat are ineffective control measures. We use a one dimensional PDE model to examine the contribution to the spatial spread rate by the size of the susceptible poultry birds, the diffusion rates of the wild birds and the virus. We notice the diffusion rate of the wild birds with high mortality has very little impact on the spread speed. But for the wild birds who can survive the infection, depending on the direction of convection, their diffusion rate can substantially increase the spread rate, indicating a significant role of the migration of these type of wild birds in the spread of the disease.
\end{abstract}

Key words: Avian Influenza, H5N1, transmission, compartmental model, traveling wave, spread rate, convection

\footnotetext{
${ }^{1}$ Corresponding author. E-mail: wujh@mathstat.yorku.ca
} 
AMS subject classification: 34C60, 35K57, 92D25, 92D30

\section{Introduction}

\subsection{Classification of influenza viruses.}

Influenza viruses belong to the family Orthomyxoviridae, and are isolated from a wide range of hosts including humans, pigs, birds, horses and sea mammals [8]. Based on antigenic differences in their gene products nucleoprotein (NP) and matrix (MI), influenza viruses are classified into three types A, B and C. Avian influenza, an infectious disease of birds that is caused by influenza virus type A strains, was identified first in Italy [23]. Influenza A strains are further classified into sub-types based on two kinds of surface glycoproteins, haemagglutinin (HA) and neuraminidase (NA). Wild aquatic birds, mainly Anseriformes are primordial reservoirs of all identified $16 \mathrm{HA}$ subtypes of avian influenza $[6,31]$. However, these viruses normally do not make wild aquatic birds sick but, once transmitted to poultry species can cause serious economic losses, arising from high mortality and trade restrictions [15].

Based on the pathogenicity influenza A viruses are categorized into two distinct groups, Highly Pathogenic Avian Influenza (HPAI) and Low Pathogenic Avian Influenza (LPAI) viruses. The HAs of HPAI differ from those of LPAI influenza A viruses by virtue of possessing multiple basic amino acids at the carboxyl terminus of HA1. This structural feature permits cellular ubiquitous proteases, to cleave the HA and render the virus infectious and able to spread to a variety of organs, leading to systemic infection [24]. By contrast, LPAI-virus HAs lack a series of basic amino acids at the cleavage site and are cleaved only by proteases secreted from cells in the respiratory or intestinal tract, or both, so that the viruses only produce localized infections, resulting in mild or asymptomatic infections [10]. Avian origin-H5N1 strains, commonly called HAPI A (H5N1) are primarily isolated from a poultry farm of Scotland, UK during 1959 [21]. Unpredictable and rapid assortment ability of virus boosted its continuous evolution, spreading to different continents since 1996-Asian outbreak. This strain acquired an unusual quality of cross species barrier, creating a great pandemic fear globally [28].

\subsection{Transmission cycle of $\mathbf{H 5 N} 1$}

Diversified adaptations of H5N1 subtype in systems, viz insects, birds, mammals (terrestrial and aquatic), human, and environment (water and land), lead to a complex transmission network. Figure 1 explicates, for the $\mathrm{H} 5 \mathrm{~N} 1$ subtype, some possible transmission pathways, mainly, birds to birds [18], birds to mammals [12], birds to human [5, 19, 25], birds to insects [14], human to human (Note: serological evidence of human-to-human transmission was limited to a few cases only [19], but inefficiently [3]), and environment to birds/mammals/human and vice-versa [33]. 


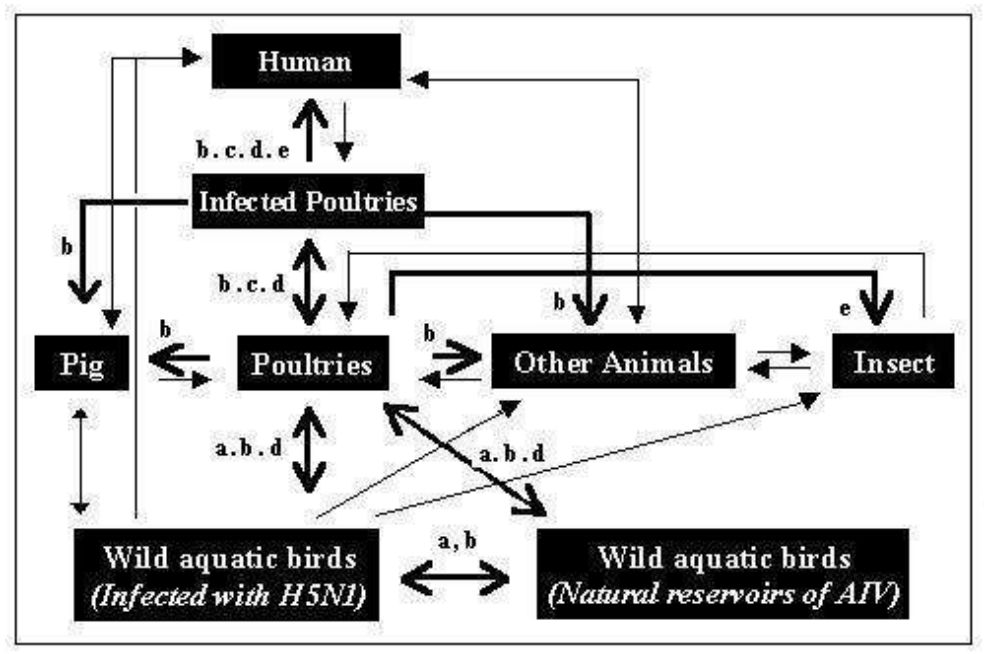

- non-confirmed routes

confirmed routes

Figure 1: The possible transmission routes of H5N1 from one host to another, through a). migration (short and long distance); b). faecal-oral route (via environment i.e., sharing of habitat); c). poultry and wild bird trade; d). contaminated feed, cages etc.; e). feeding on infected fowls.

\subsection{Modes of transmission}

The steady maintenance of $\mathrm{H} 5 \mathrm{~N} 1$ virus in various media $[13,26]$ enabled the virus to circulate from Southeast Asian countries to other parts of the world as observed in the last few years. Various transmission modes may play roles in virus hop: natural activities (bird migration, and agroecological environments) and human activities (both live and dead poultry trading [17], smuggling of wild birds [29], mechanical movement of infected materials [1] and specific farming practices.). The common diffusion routes of avian influenza are faecal-oral (H5N1 infected birds shed the virus high titers from trachea as well as from the cloacae $[9,27]$ and these are involved in the environmental contamination, where variety of hosts may get exposed and infected).

\subsection{Purposes of this study}

This study proposes a system of ordinary differential equations (ODE), which describes the transmission dynamics of $\mathrm{H} 5 \mathrm{~N} 1$ virus among the poultry, wild birds and environment. We parameterize our model using data and information from various existing literatures. We utilize the model to compare the relative effectiveness of various control measures such as culling of poultry, reducing susceptible poultry and cleaning the contaminated environment on the reduction of a key epidemiological threshold, the control reproduction number in a local outbreak. We then extend the model to incorporate some spatial aspects in order to estimate the propagation speed and to describe the spatio-temporal patterns of H5N1 spread. We start with a PDE model in one-dimensional space to see how the propagation speed (related to the minimal wave speed of the so-called traveling 
wave fronts) depends on the susceptible poultry birds, the spatial diffusion rates of poultry and wide birds, as well as the spread speed of the virus in the environment. We then carry out some intensive simulations on a corresponding two-dimensional PDE model to see how the interaction between the (random) spatial diffusion of birds and virus and the migration of wild birds affects the spatio-temporal patterns of the disease.

\section{Local outbreaks in a Homogeneous Environment}

\subsection{Basic Model Formulation}

With the above understanding of the ecology and epidemiology of $\mathrm{H} 5 \mathrm{~N} 1$, we categorize birds into three groups depending on their participation in the transmission of $\mathrm{H} 5 \mathrm{~N} 1$, as

(i) poultry (mainly chicken) (c);

(ii) wild birds (w), susceptible and die after $\mathrm{H} 5 \mathrm{~N} 1$ infection; and

(iii) wild birds (d), susceptible but survive after H5N1 infection without apparent disease symptoms.

Figure 2, a mathematical abstraction of Figure 1, depicts the flow of the transmission of the virus between birds and environment. These infected poultry birds as well as the exposed/infected wild birds may contribute to environmental contamination through the common faecal-to-oral route.

The birds groups are further divided as susceptible $\left(S_{c}, S_{w}\right.$ and $\left.S_{d}\right)$, exposed $\left(E_{w}\right)$, infected $\left(I_{c}, I_{w}\right.$ and $\left.I_{d}\right)$ and recovered $\left(R_{d}\right)$ compartments. Infected $\left(I_{c}, I_{w}\right.$, and $\left.I_{d}\right)$ class includes asymptomatic and symptomatic stages. Where as in group $(w)$, Exposed $\left(E_{w}\right)$ and Infected $\left(I_{w}\right)$ classes are defined separately since the exposed wild birds $\left(E_{w}\right)$ can fly some distances even after viral exposure, helps to describe the spatio-temporal patterns. Recovered class $(R)$ was defined only for (d) group wild birds as $\left(R_{d}\right)$ and was excluded for the remaining groups, (c) and (w) since H5N1 is fatal.

The virus can persist in the water, sewage, excreta and outside the host in suitable conditions, thus we introduce a new variable/class $\mathrm{V}$ to describe the virus density in the environment. The model formulated below incorporates direct host-host transmission, in which susceptible hosts become infected through direct fecal-oral transmission between the same groups of species birds as we divided. In addition, infected birds shed the virus into their local environment and this is reflected by including the indirect fecal-oral transmission via encounters through the contaminated environment.

The model, a nonlinear system of ordinary differential equations, is formulated to characterize the transmission dynamics of birds and virus in a local outbreak with a relatively homogeneous environment. Suppressing the time-dependence for each variable, the model is given by 


$$
\left\{\begin{array}{l}
\dot{S}_{c}=-\beta_{c} S_{c} V-\alpha_{c} S_{c} I_{c} / N_{c} \\
\dot{I}_{c}=\beta_{c} S_{c} V+\alpha_{c} S_{c} I_{c} / N_{c}-d_{i c} I_{c} \\
\dot{S}_{w}=-\beta_{w} S_{w} V-\alpha_{e w} S_{w} E_{w} / N_{w}-\alpha_{i w} S_{w} I_{w} / N_{w} \\
\dot{E}_{w}=\beta_{w} S_{w} V+\alpha_{e w} S_{w} E_{w} / N_{w}+\alpha_{i w} S_{w} I_{w} / N_{w}-\mu_{w} E_{w} \\
\dot{I_{w}}=\mu_{w} E_{w}-d_{i w} I_{w} \\
\dot{S}_{d}=-\beta_{d} S_{d} V-\alpha_{d} S_{d} I_{d} / N_{d}+\eta_{d} R_{d} \\
\dot{I}_{d}=\beta_{d} S_{d} V+\alpha_{d} S_{d} I_{d} / N_{d}-\gamma_{d} I_{d} \\
\dot{R}_{d}=\gamma_{d} I_{d}-\eta_{d} R_{d} \\
\dot{V}=r_{c} I_{c}+r_{e w} E_{w}+r_{i w} I_{w}+r_{d} I_{d}-d_{v} V-d_{n} V-\left(\delta_{c} N_{c}+\delta_{w} N_{w}+\delta_{d} N_{d}\right) V
\end{array}\right.
$$

where

$$
N_{c}=S_{c}+I_{c}, \quad N_{w}=S_{w}+E_{w}+I_{w}, \quad N_{d}=S_{d}+I_{d}+R_{d} .
$$

Precise definitions of parameters and their identification from existing references are provided in the next sub-section.

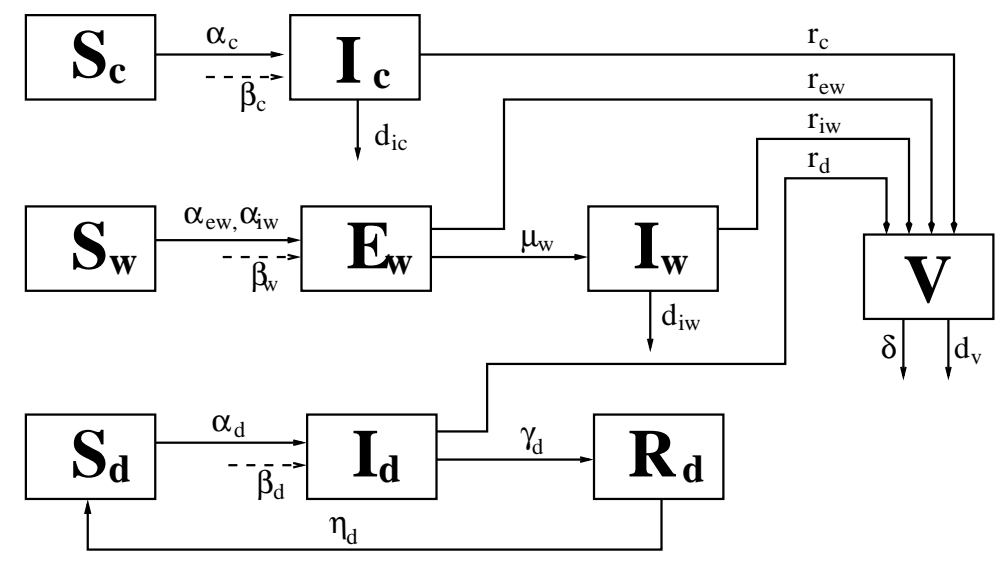

Figure 2: Flow diagram represents the transmission routes of the H5N1 virus in the interaction between different bird populations and the environment. The bird population consists of three different types that are further stratified in terms of disease progression into several compartments.

\subsection{Initial Conditions and Model Parameters}

We will use $\left(S_{c 0}, S_{w 0}, S_{d 0}\right)$ to denote the initial numbers of susceptible $c, w$ and $d$ classes of birds, and will be taken as $(20000,500,500)$ in our simulations. Note that $S_{c 0}$ can be altered in outbreak control through culling. 
Other parameters are described in Table 1. Here we provide a few remarks about the estimation and choice of certain parameter values.

The direct transmission rate $\alpha_{c}$ is the number of contacts that a susceptible poultry bird makes per day, times the probability of disease transmission for each contact between a susceptible and an infected poultry bird. Similarly, we can define $\alpha_{e d}, \alpha_{i w}$ and $\alpha_{d}$. The indirect transmission rate is defined as the number of contacts of one susceptible bird (in the respective class) with the environment per day, times the probability of disease transmission per contact per virus density.

It is still unknown how long it takes for the $d$ class birds to loss their immunity to the H5N1 virus. In our simulations, we assume recovered $d$ class of birds gain life long immunity.

In our model, $r_{d}$ is the shedding rate of infected $d$ class birds per day. This is determined from the embryo infective dose that is the titre required to infect $50 \%$ of the embryos inoculated with the virus.

All of these transmission rates (direct and/or indirect) are unknown and can be changed once some control measures are implemented. A report from China [34] shows that serological studies conducted to observe the prior infection of a total number of 1092 migratory ducks with H5N1 in China found $3.1 \%$ had antibodies of $\mathrm{H} 5 \mathrm{~N} 1$ strain. We therefore take the infected rate of the $d$ class of birds as $3.1 \%$.

We the use the above rate to estimate other parameters, under the assumption that no control measures are taken and using the values of known parameters $d_{i c}, d_{i w}, \mu_{w}, \gamma_{d}, \eta_{d}, r_{c}, r_{e w}, r_{i w}, r_{d}, d_{v}$ listed in the Table 1 , as well as the initial value

$$
\left(S_{c}, I_{c}, S_{w}, E_{w}, I_{w}, S_{d}, I_{d}, R_{d}, V\right)=(20000,0,500,0,0,500,2,0,0)
$$

(that is, we assume only 2 infected $d$ class birds at beginning). The identified values of transmission rates, reported in Table 1, give the best fitted infected rate of the $d$ class birds around $3.1 \%$.

The virus in the environment is consumed by birds and others, and can also be reduced by some human efforts. These are reflected by the consumption rates $\delta_{c}, \delta_{w}, \delta_{d}$ and $d_{n}$. We assume that $\delta_{c}, \delta_{w}$ and $\delta_{d}$ are relatively small, and we will vary $d_{n}$ to see the effectiveness of cleaning the environment in a local outbreak control.

\subsection{Effectiveness of Some Control Measures in a Local Outbreak}

Of critical importance of the outbreak control is a threshold which determines whether or not the disease can invade successfully. This is called the basic reproduction number $\mathcal{R}_{0}$, defined to be the expected number of secondary cases produced, in a completely susceptible population, by a typical infected individual during its entire period of infectiousness. In our case, since some of the parameter values can be changed when certain control measures such as culling of domestic birds, we shall call this threshold "control reproduction number". In deterministic models, control is possible when and only when $\mathcal{R}_{0}<1$ [7].

We can use the well-known approach developed in [30] to calculate $\mathcal{R}_{0}$. First of all, we note that the disease free state is

$$
E_{0}\left(S_{c}, I_{c}, S_{w}, E_{w}, I_{w}, S_{d}, I_{d}, R_{d}, V\right)=\left(S_{c 0}, 0, S_{w 0}, 0,0, S_{d 0}, 0,0,0\right),
$$


Table 1: Model parameters: definitions, estimations and assignment (unit: per day)

\begin{tabular}{|c|c|c|c|}
\hline Parameter & Definition & Value & Reference \\
\hline$\alpha_{c}$ & $\begin{array}{l}\text { Direct transmission rate between } \\
\text { poultry (per day per poultry) }\end{array}$ & 0.03 & \\
\hline$\alpha_{e w}$ & $\begin{array}{l}\text { Direct transmission rate between } \\
S_{w} \text { and } E_{w} \text { (per day per bird) }\end{array}$ & 0.0001 & \\
\hline$\alpha_{i w}$ & $\begin{array}{l}\text { Direct transmission rate between } \\
S_{w} \text { and } I_{w} \text { (per day per bird) }\end{array}$ & 0.0002 & \\
\hline$\alpha_{d}$ & $\begin{array}{l}\text { Direct transmission rate between } d \\
\text { class birds (per day per bird) }\end{array}$ & 0.0002 & \\
\hline$\beta_{c}$ & $\begin{array}{l}\text { Indirect transmission rate } \\
\text { ( per virus density) }\end{array}$ & $4 * 10^{-16}$ & \\
\hline$\beta_{w}$ & $\begin{array}{l}\text { Indirect transmission rate } \\
\text { ( per virus density } t \text { ) }\end{array}$ & $4 * 10^{-16}$ & \\
\hline$\beta_{d}$ & $\begin{array}{l}\text { Indirect transmission rate } \\
\text { ( per virus density) }\end{array}$ & $4 * 10^{-16}$ & \\
\hline$d_{i c}$ & Disease induced death rate of infected poultry & $1 / 3(1 / 2.6-1 / 3.9)$ & {$[34]$} \\
\hline$d_{i w}$ & $\begin{array}{l}\text { Disease induced death rate of } \\
\text { infected } w \text { class birds }\end{array}$ & $1 / 5$ & [34] \\
\hline$\mu_{w}$ & Transmission rate from exposed to infected & $0.2(1 / 3-1 / 7)$ & {$[4]$} \\
\hline$\gamma_{d}$ & Recovery rate of infected $d$ class birds & $0.05(1 / 14-1 / 28)$ & {$[33]$} \\
\hline$\eta_{d}$ & $\begin{array}{l}\text { Rate at which } d \text { class } \\
\text { birds loss their immunity }\end{array}$ & 0 & \\
\hline$r_{c}$ & Shedding rate of infected poultry & $10^{10} \mathrm{EID}_{50}^{4}$ & {$[33]$} \\
\hline$r_{e w}$ & Shedding rate of exposed $w$ class birds & $10^{10} \mathrm{EID}_{50}^{4}$ & {$[33]$} \\
\hline$r_{i w}$ & Shedding rate of infected $w$ class birds & $10^{10} \mathrm{EID}_{50}^{4}$ & [33] \\
\hline$r_{d}$ & Shedding rate of infected $d$ class birds & $10^{10} \mathrm{EID}_{50}^{4}$ & {$[33]$} \\
\hline$d_{v}$ & Death rate of the H5N1 virus (per day) & $0.1(1 / 17-)$ & $\begin{array}{l}{[26]} \\
{[32]}\end{array}$ \\
\hline$\delta_{c}$ & $\begin{array}{l}\text { Consumption rate of the virus in the } \\
\text { environment (per day, per poultry bird) }\end{array}$ & $10^{-15}$ & see text \\
\hline$\delta_{w}$ & $\begin{array}{l}\text { Consumption rate of the virus in the } \\
\text { environment (per day, per } w \text { bird) }\end{array}$ & $10^{-15}$ & see text \\
\hline$\delta_{d}$ & $\begin{array}{l}\text { Consumption rate of the virus in the } \\
\text { environment (per day, per } c \text { bird) }\end{array}$ & $10^{-15}$ & see text \\
\hline$d_{n}$ & $\begin{array}{l}\text { Consumption (by others) rate of } \\
\text { the virus in the environment (per day) }\end{array}$ & see text & see text \\
\hline
\end{tabular}


where $S_{c 0}, S_{w 0}, S_{d 0}$ are initial conditions. We then calculate the corresponding Jacobian matrix, $\mathcal{F}$ and $\mathcal{V}$, describing the linearization of a reduced system about the disease free state $E_{0}$, yielding

$$
\mathcal{F}=\left(\begin{array}{ccccc}
\alpha_{c} & 0 & 0 & 0 & \beta_{c} S_{c 0} \\
0 & \alpha_{e w} & \alpha_{i w} & 0 & \beta_{w} S_{w 0} \\
0 & 0 & 0 & 0 & 0 \\
0 & 0 & 0 & \alpha_{d} & \beta_{d} S_{d 0} \\
r_{c} & r_{e w} & r_{i w} & r_{d} & 0
\end{array}\right), \mathcal{V}=\left(\begin{array}{ccccc}
d_{i c} & 0 & 0 & 0 & 0 \\
0 & \mu_{w} & 0 & 0 & 0 \\
0 & -\mu_{w} & d_{w} & 0 & 0 \\
0 & 0 & 0 & \gamma_{d} & 0 \\
0 & 0 & 0 & 0 & d_{v}+d_{n}+\delta_{0}
\end{array}\right)
$$

where $\delta_{0}=\delta_{c} S_{c_{0}}+\delta_{w} S_{w 0}+\delta_{d} S_{d 0}$. Thus, the basic reproduction number of model (2.1) is given by

$$
\mathcal{R}_{0}=\rho\left(\mathcal{F} \mathcal{V}^{-1}\right)
$$

where $\rho$ is the spectral radius of a matrix. Unfortunately, the explicit expression of the basic reproduction number $\mathcal{R}_{0}$ is too complicated for the general model to have any practical use. In the following part of this subsection, we will investigate $\mathcal{R}_{0}$ by plotting it against some parameters of epidemiological interests or of importance to guide the design of control measures.

We now present some numerical simulations to illustrate and quantify effectiveness of some feasible control measures on the basic reproduction number $\mathcal{R}_{0}$ that, as shown in (2), is a function of the initial host population sizes, transmission rates, disease-induced death rates, and the virus' shedding and consumption rates.

The initial susceptible poultry population can be controlled by (indiscriminatively) culling or by keeping the highly susceptible chickens in bird-proof premises that are far away from open waters on which virus aggregate. Figure 3 (a) clearly shows the significance of reducing the susceptible poultry population, since $\mathcal{R}_{0}$ increases sharply as $S_{c 0}$ (number of initial susceptible poultry birds) increases. Using parameters from Table 1, a local outbreak can be controlled if the initial poultry population size is reduced from $2 \times 10^{4}$ to $0.1 \times 10^{4}$ (in other words, culling $95 \%$ of poultry population can reduce the reproduction number to below 1).

Figure 3 (b) plots $\mathcal{R}_{0}$ as a function of the death rate of infected poultry, i.e., the life span of infected poultry. Note that this rate is the sum of the death rates of the poultry induced by the disease and by other control measures such as culling. The thick part in the graph corresponds to the observed life span of infected chicken without human intervention, and this yields the value of $\mathcal{R}_{0}$ above 3.3 under the set of parameters we selected in Table 1, which shows the disease will persist without other human interventions. This figure also shows, again using the parameters from Table 1, that control of a local outbreak is possible if the life span of infected poultry can be decreased to less than one day. Therefore, effective and speedy culling can control a local outbreak.

In Figure 3 (c), $\mathcal{R}_{0}$ is plotted as function of $d_{n}$, the efficacy of control measure of cleaning environment in a local outbreak control. We can observe if the virus can be killed more than $40 \%$ when the environment is cleaned, the local outbreak can be prevented. As such, cleaning the environment seems to be another control measure.

In Figure $3(d), \mathcal{R}_{0}$ is plotted as function of initial number of susceptible wild $d$ class birds. From this graph, we can see if we decrease the number of susceptible wild birds, the basic reproduction number just decreases slightly but still much greater than one. This shows that culling wild birds and destroying their habitat are ineffective control measures, which coincide with the 
conclusion reached in the Scientific Seminar on Avian Influenza, the Environment and Migratory Birds, April 10-11, 2006, at UN Office in Nairobi, Kenya.

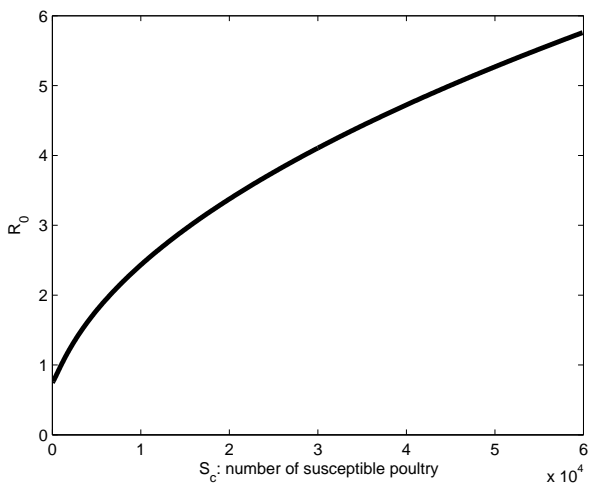

(a) $\mathcal{R}_{0}$ plotted against $S_{c 0}$

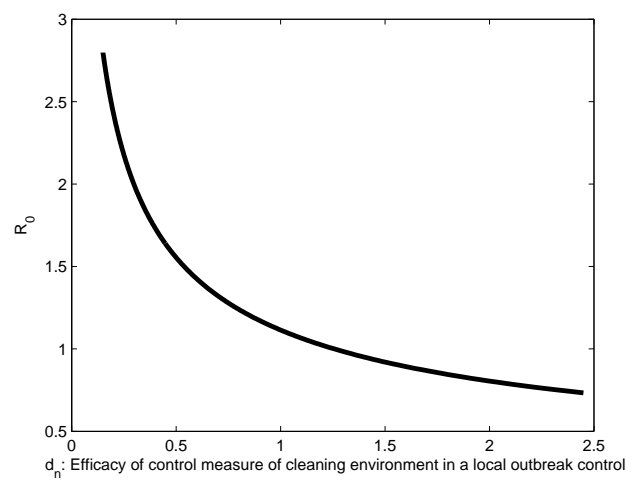

(c) $\mathcal{R}_{0}$ plotted against $d_{v}$

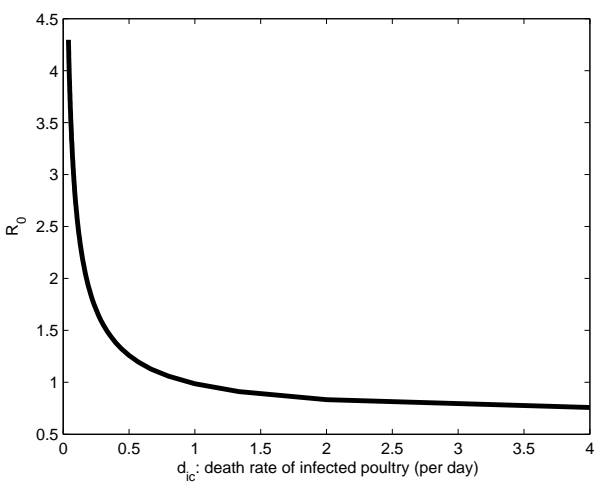

(b) $\mathcal{R}_{0}$ plotted against $d_{i c}$

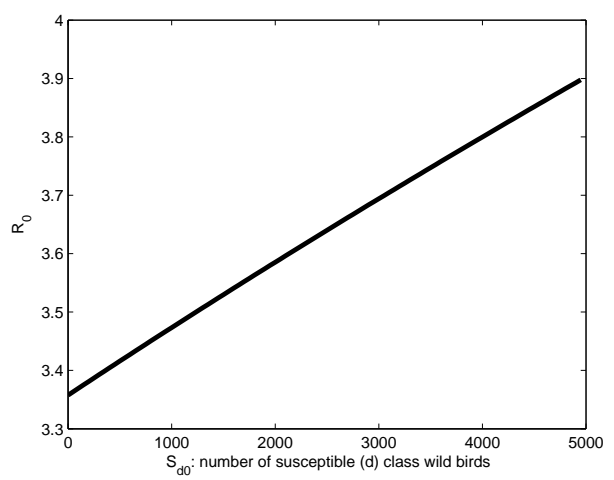

(d) $\mathcal{R}_{0}$ plotted against $S_{d}$

Figure 3: Plots of $\mathcal{R}_{0}$ as a function of the susceptible poultry population size, the death rates of the poultry birds, the death rate of the virus in the environment and the initial susceptible wild birds.

\section{Impact of the Mobility of Birds and Virus}

As discussed above, the domestic waterfowl, specific farming practices and agro-ecological environments played key roles in the occurrence, maintenance, and spread of the H5N1 virus for many affected countries. Although numerous wild birds have also become infected, it has been much debated whether they played an active role in the geographic spread of the disease. It has been argued that infected birds would be too severely affected to continue their flight, this consequence might hamper the bird's migration and movement and thus unlikely to spread the H5N1 virus. On the other hand, it has been argued that although this may be true for some wild birds, it has been shown that, in some experiments, several birds species survive after infection and shed the H5N1 
virus without apparent disease signs [5]. It is thus important to study how the spread speed of the disease depends on parameters such as the recovery rate of infected wild birds, the shedding rates of all kinds of infected birds, and the diffusion rates of birds in all categories using a suitable PDE model.

\subsection{ODE Model Simplification}

We start with a simplification of the ODE model in order to further analyze the impact of the mobility of birds and virus on the spread speed of the disease.

Denote $N_{d}=S_{d}+I_{d}+R_{d}$ as the total number $d$ class birds. Adding the equations about $S_{d}, I_{d}, R_{d}$ in model (2.1) together, we have $\dot{N}_{d}=0$. So $N_{d}(t)=N_{d}$ is constant for all $t \geq 0$. If we further assume the recovered $d$ class birds become susceptible immediately, i.e., $\eta_{d} \rightarrow \infty$ (this assumption of immediate return to the susceptible class may slightly overestimate the rate of disease progression), then the equation for $R_{d}$ in model (2.1) can be solved in terms of $I_{d}$. With $R_{d}(0)=0$, we have

$$
R_{d}(t)=e^{-\eta_{d} t} \int_{0}^{t} \gamma_{d} I_{d}(\tau) e^{-\eta_{d} \tau} d \tau .
$$

Thus, $R_{d}(t) \rightarrow 0$ as $\eta_{d} \rightarrow \infty$. Therefore, $N_{d}=S_{d}+I_{d}$, and we need consider only the equation for infectious $d$ class birds, which takes the form

$$
\dot{I}_{d}=\beta_{d}\left(N_{d}-I_{d}\right) V+\alpha_{d}\left(N_{d}-I_{d}\right) I_{d}-\gamma_{d} I_{d} .
$$

Then the simplified model takes the forms as

$$
\left\{\begin{array}{l}
\dot{S}_{c}=-\beta_{c} S_{c} V-\alpha_{c} S_{c} I_{c} / N_{c} \\
\dot{I}_{c}=\beta_{c} S_{c} V+\alpha_{c} S_{c} I_{c} / N_{c}-d_{i c} I_{c} \\
\dot{S_{w}}=-\beta_{w} S_{w} V-\alpha_{e w} S_{w} E_{w} / N_{w}-\alpha_{i w} S_{w} I_{w} / N_{w} \\
\dot{E_{w}}=\beta_{w} S_{w} V+\alpha_{e w} S_{w} E_{w} / N_{w}+\alpha_{i w} S_{w} I_{w} / N_{w}-\mu_{w} E_{w} \\
\dot{I_{w}}=\mu_{w} E_{w}-d_{i w} I_{w} \\
\dot{I_{d}}=\beta_{d}\left(N_{d}-I_{d}\right) V+\alpha_{d}\left(N_{d}-I_{d}\right) I_{d} / N_{d}-\gamma_{d} I_{d} \\
\dot{V}=r_{c} I_{c}+r_{e w} E_{w}+r_{i w} I_{w}+r_{d} I_{d}-\left(d_{v}+d_{n}\right) V-\left(\delta_{c} N_{c}+\delta_{w} N_{w}+\delta_{d} N_{d}\right) V
\end{array}\right.
$$

If we assume $N_{d}=S_{d 0}$, the simplified model (3.3) and the original model (2.1) have the same disease free equilibrium $E_{0}$, so is the basic reproduction number $\mathcal{R}_{0}$. When $\mathcal{R}_{0}>1$, there is a local outbreak, and as $t \rightarrow \infty$,

$$
S_{c}(t) \rightarrow S_{c, \infty}, \quad S_{w} \rightarrow S_{w, \infty}
$$

for two constants $S_{c, \infty}$ and $S_{w, \infty}$, and $I_{c}(t), E_{w}(t), I_{w}(t), T_{d}(t), V(t) \rightarrow 0$ [2]. 


\subsection{Spread Speed}

We now incorporate the spatial content to the simplified model (3.3) and obtain the following PDE model for which each variable is a function of both the spatial location $x \in R$ and the time $t \in R$ :

$$
\left\{\begin{aligned}
\frac{\partial S_{c}}{\partial t}= & -\beta_{c} S_{c} V-\alpha_{c} S_{c} I_{c} / N_{c}-v_{c} \frac{\partial S_{c}}{\partial x}+D_{c} \frac{\partial^{2} S_{c}}{\partial x^{2}} \\
\frac{\partial I_{c}}{\partial t}= & -v_{w} \frac{\partial S_{w}}{\partial x} \beta_{c} S_{c} V+\alpha_{c} S_{c} I_{c} / N_{c}-d_{i c} I_{c}-v_{c} \frac{\partial I_{c}}{\partial x} \\
\frac{\partial S_{w}}{\partial t}= & -\beta_{w} S_{w} V-\alpha_{e w} S_{w} E_{w} / N_{w}-\alpha_{i w} S_{w} I_{w} / N_{w}-v_{w} \frac{\partial S_{w}}{\partial x}+D_{w} \frac{\partial^{2} S_{w}}{\partial x^{2}} \\
\frac{\partial E_{w}}{\partial t}= & \beta_{w} S_{w} V+\alpha_{e w} S_{w} E_{w} / N_{w}+\alpha_{i w} S_{w} I_{w} / N_{w}-\mu_{w} E_{w}-v_{w} \frac{\partial E_{w}}{\partial x}+D_{w} \frac{\partial^{2} E_{w}}{\partial x^{2}} \\
\frac{\partial I_{w}}{\partial t}= & \mu_{w} E_{w}-d_{i w} I_{w}, \\
\frac{\partial I_{d}}{\partial t}= & \beta_{d}\left(N_{d}-I_{d}\right) V+\alpha_{d}\left(N_{d}-I_{d}\right) I_{d} / N_{d}-\gamma_{d} I_{d}-v_{d} \frac{\partial I_{d}}{\partial x}+D_{d} \frac{\partial^{2} I_{d}}{\partial x^{2}} \\
\frac{\partial V}{\partial t}= & r_{c} I_{c}+r_{e w} E_{w}+r_{i w} I_{w}+r_{d} I_{d}-\left(d_{v}+d_{n}\right) V \\
& -\left(\delta_{c} N_{c}+\delta_{w} N_{w}+\delta_{d} N_{d}\right) V-v_{v} \frac{\partial V}{\partial x}+D_{v} \frac{\partial^{2} V}{\partial x^{2}}
\end{aligned}\right.
$$

where $D_{c}\left(v_{c}\right), D_{w}\left(v_{w}\right), D_{d}\left(v_{d}\right)$ and $D_{v}\left(v_{v}\right)$ are the diffusion (convection) coefficients of the susceptible poultry birds, susceptible and exposed $w$ class birds, infected $d$ class birds and the virus in the environment.

Normally, the mobility of the poultry birds is rather limited, comparing with free-living birds. So $D_{c}<<1$ is quite small, with respect to $D_{w}$ and $D_{d}$. We include the spatial movement of the exposed but ignore the infected $w$ class birds. As the H5N1 virus in the environment can move due to the floating water and air or due to the transportation, we also allow a spatial diffusion term for the variable $v$.

The PDE model (3.4) has the so-called traveling wave fronts: a special type of solutions depending only on the wave variable $z=x+c t$ with the wave speed $c \geq c_{\min }>0$, and connecting the disease free equilibrium $\left(S_{c 0}, 0, S_{w 0}, 0,0,0,0\right)$ (at the initial stage) to the disease free equilibrium $\left(S_{c, \infty}, 0, S_{w, \infty}, 0,0,0,0\right)$ (after the epidemic). In principle, we anticipate that solutions of the PDE model (3.4) converges to the traveling wave with the minimal wave speed $c_{\min }$ that coincides with the propagation speed of the disease [20], and our simulations do support this anticipation.

It is therefore critical to calculate the minimal wave speed. For this purpose, we linearise the equations for $I_{c}, E_{w}, I_{w}, I_{d}$, and $V$ in the region $x \rightarrow-\infty$ where $S_{c} \rightarrow S_{c 0}, S_{w} \rightarrow S_{w 0}, S_{d} \rightarrow$ 
$N_{d}$ and the remaining variables approach zero. The linearised equations are then converted to travelling wave form by looking for solutions that are functions of only the wave variable $z=x+c t$ with $c \geq 0$. Then we look for non-trivial solutions of the linearised travelling wave equations of the form $\left(I_{c}, E_{w}, I_{w}, I_{d}, V\right)=\left(q_{1}, q_{2}, q_{3}, q_{4}, q_{5}\right) \exp (\lambda z)$. After a fair amount of algebra we find that the characteristic matrix

$$
C(\lambda ; c)=\left(\begin{array}{ccccc}
a_{11} & & & & \beta_{c} S_{c 0} \\
& a_{22} & \alpha_{i w} & & \beta_{w} S_{w 0} \\
& \mu_{w} & -d_{i w}-c \lambda & & \\
& & & a_{44} & \beta_{d} N_{d} \\
r_{c} & r_{e w} & r_{i w} & r_{d} & a_{55}
\end{array}\right),
$$

where

$$
\begin{aligned}
& a_{11}=\alpha_{c}-d_{i c}-c \lambda-v_{c} \lambda \\
& a_{22}=\alpha_{e w}-\mu_{w}-c \lambda-v_{w} \lambda+D_{w} \lambda^{2} \\
& a_{44}=\alpha_{d}-\gamma_{d}-c \lambda-v_{d} \lambda+D_{d} \lambda^{2} \\
& a_{55}=-d_{v}-c \lambda-v_{v} \lambda+D_{v} \lambda^{2}-\left(\beta_{c} S_{c 0}+\beta_{w} S_{w 0}+\beta_{d} N_{d}\right) .
\end{aligned}
$$

Then the characteristic equation can be written as

$$
\operatorname{det} C(\lambda ; c)=0
$$

An epidemiologically feasible wave-front is one in which all the variables remain non-negative as $x \rightarrow-\infty$ (as $z \rightarrow-\infty$ in the travelling wave variable formulation). The decay of $I_{c}, E_{w}, I_{w}, I_{d}$, and $V$ to zero as $z \rightarrow-\infty$ must not be oscillatory. It is therefore necessary that there should exist at least one strictly positive real root $\lambda$ of the characteristic equation (3.5) with the property that the corresponding eigenvector $\left(q_{1}, q_{2}, q_{3}, q_{4}, q_{5}\right)$ points into the positive octant in $\mathbf{R}^{5}$. This actually happens only for $c$ above some minimum value $c_{\min }>0$.

The minimum speed $c_{\min }$ depends on the values of the parameters in a complicated way, but numerically it can be found by solving the simultaneous equations

$$
\begin{aligned}
\operatorname{det} C(\lambda ; c) & =0, \\
\frac{d \operatorname{det} C(\lambda ; c)}{d \lambda} & =0,
\end{aligned}
$$

for $c$ and $\lambda$ with $c>0$ and $\lambda>0$.

Figure 4 gives the numerical solution of the minimum speed $c_{\min }$ calculated from (3.6) versus the initial number of susceptible poultry $S_{c 0}$ corresponding to different convection velocity of poultry $v_{c}$ (Figure 4 (a)); versus the diffusion coefficient of wild birds dying from the infection(Figure 4(b)); versus the diffusion coefficient of wild birds surviving of the infection (Figure 4(c)); and versus the diffusion coefficient of virus (Figure 4 (d)).

From Figure 4 (a), it is clear that for every convection velocity $v_{c}$, there exists a threshold of the initial number of susceptible poultry below which the spread rate is almost linearly dependent on the initial number of susceptible poultry, but above which the size of the initial number of poultry has almost no impact on the spread speed $c_{\min }$. Note also that the spread rate is also an increasing function of the convection velocity $v_{c}$. 


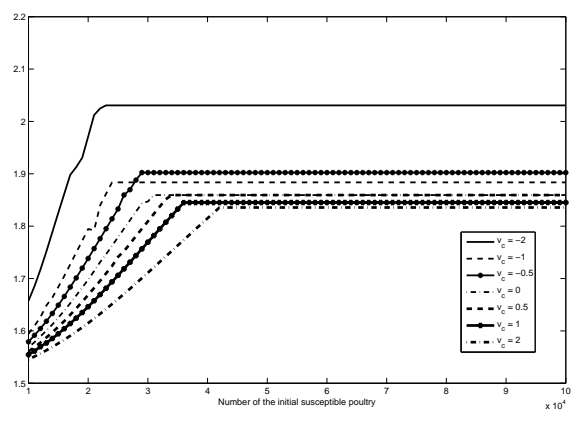

(a) Minimum speed $c_{\min }$ as function of $S_{c 0}$

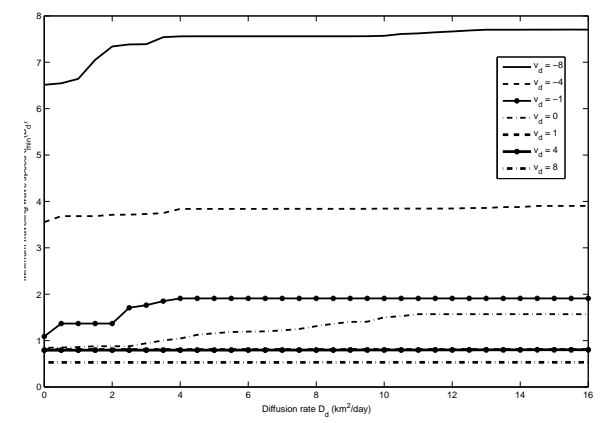

(c) Minimum speed $c_{\min }$ as function of $D_{d}$

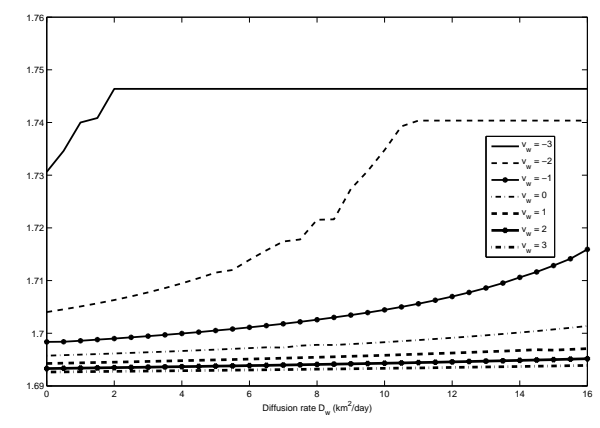

(b) Minimum speed $c_{\min }$ as function of $D_{w}$

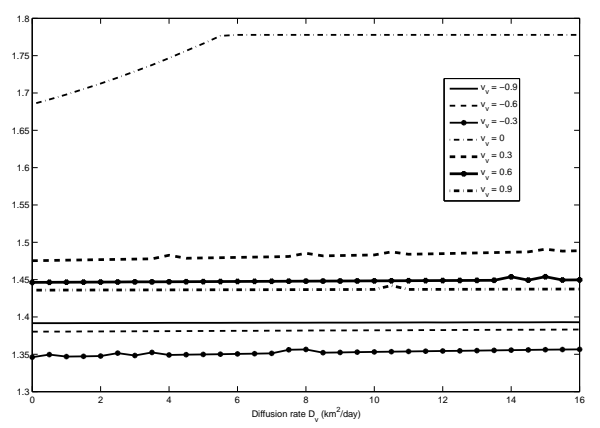

(d) Minimum speed $c_{\min }$ as function of $D_{v}$

Figure 4: The disease propagation speed $c_{\min }$ as a function of parameter $S_{c 0}, D_{w}, D_{d}$, and $D_{v}$, respectively. In each subplot, except the the parameter taken as a variable, we fix all other parameters as shown in Table 1. The spread speed is $1.2 \mathrm{~km} /$ day when all parameters are fixed as in Table 1, and when $D_{w}=9 \mathrm{~km}^{2} /$ day, $D_{d}=10 \mathrm{~km}^{2} /$ day and $D_{v}=1 \mathrm{~km}^{2} /$ day.

The diffusion rate $D_{w}$ has very little impact on the spread speed, as the variation of the minimal wave speed is between 1.69 and $1.74 \mathrm{~km} /$ day for a wide range of diffusion rates $D_{w}$ and convection velocities $v_{w}$. This seems to support the conclusion that random movement of the $w$ class birds has contributed less to the disease spread since they lose their ability to fly, once they become infectious.

Interesting phenomena can be observed in Figure 4 (c): depending on the direction of convection, the spread rate can be dramatically changed, increasing from about $2 \mathrm{~km} /$ day to $7.8 \mathrm{~km} /$ day. This indicates the migration of $d$ class birds may play a key role for the spread of the disease.

The diffusion rate of the virus depends on many factors such as the wind and the transportation tools for poultry. Figure 4 (d) shows the diffusion rate of the virus has limited effect on the spread spread. 


\section{Effects of commercial poultry transportation}

Generally, poultry birds live in restricted environments, except during the process of trading. Wild birds travel seasonally along the migratory pathways. Some studies about the virus survivability and stability in the water bodies indicate that water bodies might play a key role in the spread of the H5N1 virus. To qualitatively explore how these facts affect the spread of the virus, we need to expand the model to a two-dimensional geographical region to account for different directions of the convection of seasonal migration of wild birds. We report here some preliminary numerical analysis based on the following partial differential equations model:

$$
\left\{\begin{aligned}
\frac{\partial S_{c}}{\partial t}= & -\beta_{c} S_{c} V-\alpha_{c} S_{c} I_{c} / N_{c}-\epsilon_{X c} v_{c} \frac{\partial S_{c}}{\partial x}-\epsilon_{Y c} v_{c} \frac{\partial S_{c}}{\partial y}+D_{c} \frac{\partial^{2} S_{c}}{\partial x^{2}}+D_{c} \frac{\partial^{2} S_{c}}{\partial y^{2}} \\
\frac{\partial I_{c}}{\partial t}= & \beta_{c} S_{c} V+\alpha_{c} S_{c} I_{c}-d_{i c} I_{c} / N_{c}-\epsilon_{X c} v_{c} \frac{\partial I_{c}}{\partial x}-\epsilon_{Y c} v_{c} \frac{\partial I_{c}}{\partial y} \\
\frac{\partial S_{w}}{\partial t}= & -\beta_{w} S_{w} V-\alpha_{e w} S_{w} E_{w} / N_{w}-\alpha_{i w} S_{w} I_{w} / N_{w} \\
& -\epsilon_{X w} v_{w} \frac{\partial S_{w}}{\partial x}-\epsilon_{Y w} v_{w} \frac{\partial S_{w}}{\partial y}+D_{w} \frac{\partial^{2} S_{w}}{\partial x^{2}}+D_{w} \frac{\partial^{2} S_{w}}{\partial y^{2}} \\
\frac{\partial E_{w}}{\partial t}= & \beta_{w} S_{w} V+\alpha_{e w} S_{w} E_{w} / N_{w}+\alpha_{i w} S_{w} I_{w} / N_{w}-\mu_{w} E_{w} \\
& -\epsilon_{X w} v_{w} \frac{\partial E_{w}}{\partial x}-\epsilon_{Y w} v_{w} \frac{\partial E_{w}}{\partial y}+D_{w} \frac{\partial^{2} E_{w}}{\partial x^{2}}+D_{w} \frac{\partial^{2} E_{w}}{\partial y^{2}} \\
\frac{\partial I_{w}}{\partial t}= & \mu_{w} E_{w}-d_{i w} I_{w}, \\
\frac{\partial I_{d}}{\partial t}= & \beta_{d}\left(N_{d}-I_{d}\right) V+\alpha_{d}\left(N_{d}-I_{d}\right) I_{d} / N_{d}-\gamma_{d} I_{d} \\
& -\epsilon_{X d} v_{d} \frac{\partial I_{d}}{\partial x}-\epsilon_{Y d} v_{d} \frac{\partial I_{d}}{\partial y}+D_{d} \frac{\partial^{2} I_{d}}{\partial x^{2}}+D_{d} \frac{\partial^{2} I_{d}}{\partial y^{2}} \\
\frac{\partial V}{\partial t}= & r_{c} I_{c}+r_{e w} E_{w}+r_{i w} I_{w}+r_{d} I_{d}-\left(d_{v}+d_{n}\right) V-\left(\delta_{c} N_{c}+\delta_{w} N_{w}+\delta_{d} N_{d}\right) V \\
& -\epsilon_{X v} v_{V} \frac{\partial V}{\partial x}-\epsilon_{Y v} v_{V} \frac{\partial V}{\partial y}+D_{v} \frac{\partial^{2} V}{\partial x^{2}}+D_{v} \frac{\partial^{2} V}{\partial y^{2}} \\
& =
\end{aligned}\right.
$$

Based on our assumptions, there is no diffusion for infected poultry and infected $w$ class birds. Taking account of the birds migration, poultry commercial transportation, virus floating along rivers and other factors, we assume $v_{c}, v_{w}, v_{d}, v_{v}$ are velocities of corresponding compartments along a direction $\left(\epsilon_{X i}, \epsilon_{Y i}\right)$ with $\epsilon_{X i}^{2}+\epsilon_{Y i}^{2}=1, i=c, w, d, v$. As infected $w$ class birds cannot move, in the equation for $I_{w}$, there is no velocity terms.

In our simulations below, we use periodic boundary conditions, i.e, the two end points of axis are connected together. In these simulations, we use backward Euler method to discrete the above 
continuous model. The initial values are homogeneous everywhere, i.e, $S_{c}=20000, I_{c}=0, S_{w}=$ $500, E_{w}=0, I_{w}=0, S_{d}=500, I_{d}=0, V=0$, except we assume at the center, there are 2 infected $d$ class wild birds. The values of parameters are taken from the Table 1 . The diffusion parameters are given as $D_{c}=0.00005, D_{w}=0.001, D_{d}=0.001, D_{v}=0.001$.

Interested readers can access to the animation based on our simulations, and here in Figure 5, we present some snapshot of the animation.

We use these snapshot to illustrate the impact on the spatial movement of the epicenter by changing the direction and magnitude of the convection velocities of various species involved. Figure 5 (a) gives the baseline scenario when there is no velocity for all species, i.e., $v_{c}=v_{w}=$ $v_{d}=v_{v}=0$. Figure 5 (b) and (c) shows how the epicenter moves dominantly along the direction of the convection of the domestic poultry (trading) and the disease spread to other direction via diffusion (random movement). Figure 5 (d) plots the case when virus move along $x$ axis, corresponding to the drifting along river or by air. Figure 5 (e) and (f) illustrates the case where $d$ class wild birds migrates along $\mathrm{x}$-direction with different velocities.

\section{Discussion}

In this paper, we proposed a basic ODE model and its PDE analogues to study the transmission dynamics and spatial spread pattern of H5N1-Avian Influenza by partitioning the birds into three classes based on their epidemiological characteristics for the disease under consideration: poultry, wild birds (susceptible and die after H5N1 infection), and wild birds (susceptible but survive after H5N1 infection without apparent disease symptoms).

We used an ordinary differential equation model (2.1) to access the effects of some control measures. We found that decreasing the number of susceptible poultry, speedy culling of infected poultry, and cleaning the environments to get rid of the virus are all effective ways to control the outbreak, but killing the wild birds with low disease mortality is ineffective.

We also expand the model to a one dimensional partial differential equation (3.4) to study the spread speed of the disease. By numerically calculating the minimum wave speed, we found that the diffusion and immigration of wild birds with low disease mortality seem to be the key factors for the spatial spread, while other factors, such as the diffusion rates and convection velocities of poultry, wild birds with high disease mortality and the virus have less impact for the propagation speed.

By constructing a two dimensional partial differential equation model (5), we examined how the epicenter moves in space and time under different assumptions about the speed and direction of poultry commercial transportation, virus drifting (along river or by air), and immigration of birds.

\section{Acknowledgement}

This work is partially supported by the Canada Research Chairs Program, Mathematics for Information Technology and Complex Systems, and the Natural Sciences and Engineering Research 
Council of Canada, and Public Health Agency of Canada. We would like to thank Hongbin Guo of York University for help with editing the paper.

\section{References}

[1] D. J. Alexander. A review of avian influenza in different bird species, Veterinary Microbiology, 74 (2000), 3-13.

[2] J. Arino, R. Jordan, P. van den Driessche. Quarantine in a multi-species epidemic model with spatial dynamics. Mathematical Biosciences, 206 (2007) No. 1, 46-60.

[3] The Writing Committee of the World Health Organization (WHO) Consultation on Human Influenza A/H5. Avian influenza A (H5N1) infection in humans. N Engl. J. Med., 353 (2005), 1374-1385.

[4] Center for Infectious Disease Research and Policy, University of Minnesota avian influenza (bird flu): Agricultural and wildlife considerations, 2006. http://www.cidrap.umn.edu/idsa/influenza/avianflu/biofacts/avflu.html.

[5] E. J. Claas, A. E. Osterhaus, R. Van Beek, J. C. De Jong, G. F. Rimmelzwaan, D. A. Senne, S. Krauss, K. F. Shortridge, R. G. Webster. Human influenza a h5n1 virus related to a highly pathogenic avian influenza virus. Lancet 351 (1998), 472-477.

[6] R. A. M. Fouchier, V. Munster, A. Wallensten, T. M. Bestebroer, S. Herfst, D. Smith, G. F. Rimmelzwaan, B. Olsen, A. D. Osterhaus. Characterization of a novel influenza a virus hemagglutinin subtype (H16) obtained from black-headed gulls. Journal of Virology, 79 (2005) 2814-2822.

[7] W. H. Hethcote. The mathematics of infectious diseases. SIAM Review, 42 (2000), No. 4, 599-653.

[8] T. Horimoto, K. Yoshihiro. Pandemic threat posed by avian influenza a viruses. Clinical microbiology reviews, (2001), 129-149.

[9] D. J. Hulse-Post, K. M. Sturm-Ramirez, J. Humberd, P. Seiler, E. A. Govorkova, S. Krauss, C. Scholtissek, P. Puthavathana, C. Buranathai, T. D. Nguyen, H. T. Long, T. S. P. Naipospos, H. Chen, T. M. Ellis, Y. Guan, J. S. M. Peiris, R. G. Webster. Role of domestic ducks in the propagation and biological evolution of highly pathogenic H5N1 influenza viruses in Asia. Proceedings of the National Academy of Sciences of the United States of America, 102 (2005), No. 30, 10682-10687.

[10] T. Ito, H. Goto, E. Yamamoto, H. Tanaka, M. Takeuchi, M. Kuwayama, Y. Kawaoka, K. Otsuki. Generation of a highly pathogenic avian influenza A virus from an avirulent field isolate by passaging in chickens. J Virol., 75 (2001), No. 9, 4439-4443. 
[11] International Institute for Sustainable Development. A summary report of the scientific seminar on avian influenza, the environment and migratory birds. Avian Influenza and Wild Birds Bulletin 123 (2006), No. 1.

[12] J. Keawcharoen, K. Oraveerakul, T. Kuiken, Ron A.M. Fouchier, A. Amonsin, S. Payungporn, S. Noppornpanth, S. Wattanodorn, A. Theamboonlers, R. Tantilertcharoen, R. Pattanarangsan, N. Arya, P. Ratanakorn, Albert D.M.E. Osterhaus, Y. Poovorawan. Avian influenza h5n1 in tigers and leopards, Emerg Infect Dis., 10 (2004), No. 12, 2189-2191.

[13] T. Kuiken, R. Guus, R. van Debby, A. van Geert, B. Marianne, F. Ron, O. Albert. Avian H5N1 influenza in cats. Science, 8 (2004), 241.

[14] S. Kyoko, H. Keita, I. Haruhiko, S. Toshinori, H. Toshihiko, T. Yoshio, K. Hiromu, T. Kiyoshi, H. Akitoyo, S. Takehiko, A. Yamada, K. Mutsuo. Detection and isolation of highly pathogenic h5nl avian influenza a viruses from blow flies collected in the vicinity of an infected poultry farm in Kyoto. Japan, Am. J. Trop. Med. Hyg., 75 (2006), No. 2, 327-332.

[15] C. W. Lee, D. L. Suarez. Application of real-time RT-PCR for the quantitation and competitive replication study of $h 5$ and $h 7$ subtype avian influenza virus. J. Virol. Methods, 119 (2004) 151-158.

[16] J.-P. Liu. Avian Influenza - a pandemic waiting to happen? J. Microbiolol. Immunol. Infect., 39 (2006), 4-10.

[17] M. Mase, M. Eto, N. Tanimura, K. Imai, K. Tsukamoto, T. Horimoto, Y. Kawaoka, S. Yamaguchi. Isolation of a genotypically unique H5N1 influenza virus from duck meat imported into Japan from China. Virology, 339 (2005), 101-109.

[18] M. Mikhail, Y. K. Nannan, R. Webster. The surface glycoproteins of H5 influenza viruses isolated from humans, chickens, and wild aquatic birds have distinguishable properties. Journal of Virology, 73 (1999), No. 2, 1146-1155.

[19] A. W. Mounts, H. Kwong, H. S. Izurieta, Y. Ho, T. Au, M. Lee, C. Buxton Bridges, S. W. Williams, K. H. Mak, J. M. Katz, W. W. Thompson, N. J. Cox, K. Fukuda. Case-control study of risk factors for avian influenza a (H5N1) disease. Hong Kong, 1997, J. Infect. Dis., 180 (1999), 505-508.

[20] J. D. Murray. Mathematical biology. Springer-Verlag, Berlin, 1989.

[21] J. C. Obenauer, J. Denson, P. K. Mehta, X. Su, S. Mukatira, D. B. Finkelstein, X. Xu, J. Wang, J. Ma, Y. Fan, K. M. Rakestraw, R. G. Webster, E. Hoffmann, S. Krauss, J. Zheng, Z. Zhang, C. W. Naeve. Large-scale sequence analysis of avian influenza isolates. Science, 311 (2006), 1576-1580.

[22] B. Olsen, V. J. Munster, A. Wallensten, J. Waldenstro, A. Osterhaus, R. Fouchier. Global patterns of influenza a virus in wild birds. Science, 312 (2006), 384-388. 
[23] E. Perroncito. Epizoozia tifoide nei gallinacei. Torino: Annali Accademia Agricoltura, 21 (1878), 87-126.

[24] R. Rott. The pathogenic determinant of influenza virus. Veterinary Microbiology, 33 (1992), 303-310.

[25] D. L. Suarez, M. L. Perdue, N. Cox, T. Rowe, C. Bender, J. Huang, D. E. Swayne. Comparisons of highly virulent h5n1 influenza a viruses isolated from humans and chickens from Hong Kong. J. Virol., 72 (1998), 6678-6688.

[26] S. Thaweesak, R. Jam-on, N. Sae-Heng, N. Meemak, D. J. Hulse-Post, K. M. Sturm-Ramirez, R. G. Webster. Domestic Ducks and H5N1 Influenza Epidemic, Thailand. Emerging infectious diseases, 12 (2006), No. 4. 575-581.

[27] K. M. Sturm-Ramirez, D. J. Hulse-Post, E. A. Govorkova, J. Humberd, P. Seiler, P. Puthavathana, C. Buranathai, T. D. Nguyen, A. Chaisingh, H. T. Long, J. S. M. Peiris, R. G. Webster. Are ducks contributing to the endemicity of highly pathogenic H5N1 influenza virus in Asia? Journal of Virology, 79 (2005), No. 17, 11269-11279.

[28] J. K. Thomas, J. Noppenberger. Avian influenza: a review. Am. J. Health Syst. Pharm., 64 (2007), No. 2, 149-165.

[29] S. van Borm, I. Thomas, G. Hanquet, B. Lambrecht, M. Boschmans, G. Dupont, M. Decaestecker, R. Scacken, B. van den Thierry. Highly pathogenic H5N1 influenza virus in smuggled Thai eagles. Belgium. Emerging Infectious Diseases, 11 (2005), No. 5, 702-705.

[30] P. Van den Driessche, J. Watmough. Reproduction numbers and sub-threshold endemic equilibria for compartmental models of disease transmission. Mathematical Biosciences, 180 (2002), 29-48.

[31] R. G. Webster, K. F. Schortidge, Y. Kowaoka. Influenza interspecies transmission and emergence of new pandemics. FEMS Immunol., Med. Microbiol., 18 (1997), 275-279.

[32] WHO. Laboratory study of H5N1 viruses in domestic ducks: main findings. World Health Organization, 10.29, 2004.

[33] WHO. Review of latest available evidence on risks to human health through potential transmission of avian influenza (H5N1) through water and sewage. World Health Organization, WHO/SDE/WSH/06.1, 2006.

[34] J. Zhou, H. Shen, H. Chen, G. Tong, M. Liao, H. Yang, J. Liu. Characterization of a highly pathogenic H5N1 influenza virus derived from bar-headed geese in China. J Gen Virol, 87 (2006), 1823-1833. 

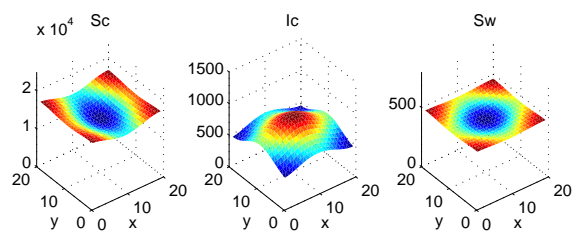

Ew

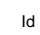

$x 10^{13} \mathrm{~V}$
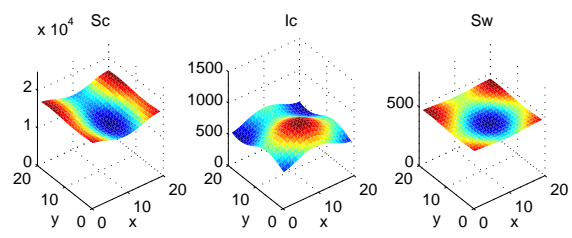

Ew

Id

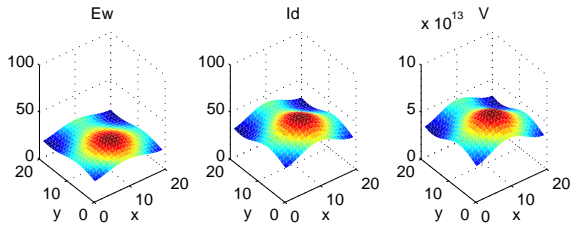

(a) no velocity

(b) $v_{c}=0.005: \mathrm{x}$ direction
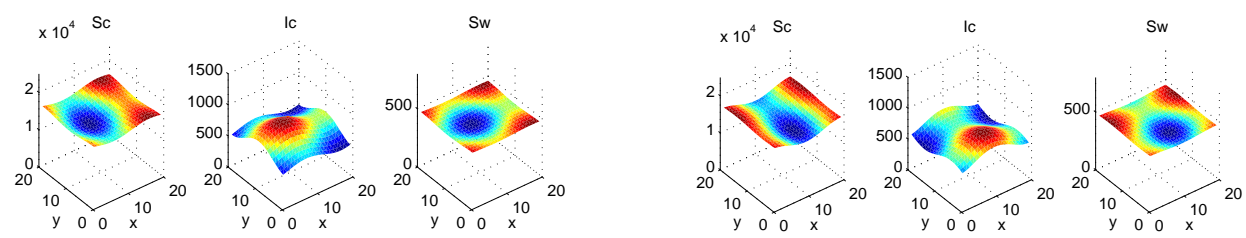

$\mathrm{Ew}$

$\times 10^{13} \mathrm{~V}$

Ew

$$
\times 10^{13} \mathrm{~V}
$$
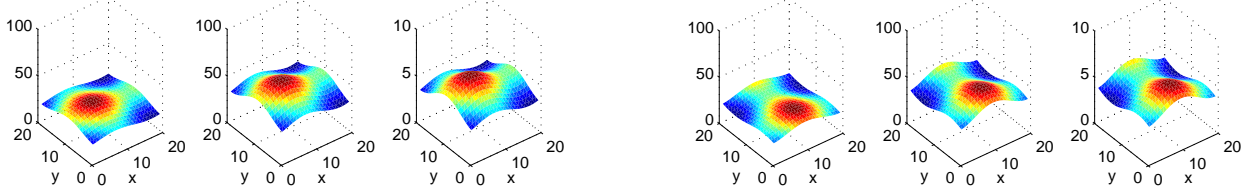

(c) $v_{c}=0.005:$ direction

(d) $v_{v}=0.005: \mathrm{x}$ direction
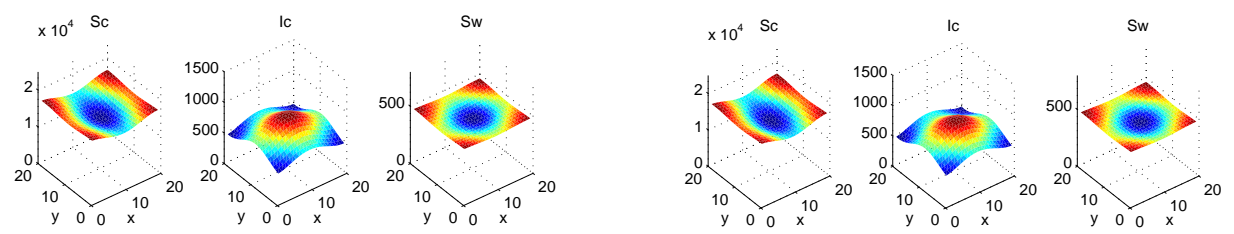

Ew

Id

$\times 10^{13} \mathrm{~V}$

Ew

Id

$\times 10^{13} \mathrm{~V}$

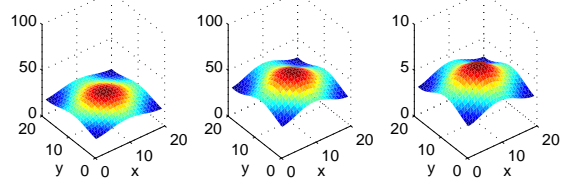

(e) $v_{d}=0.005: \mathrm{x}$ diection

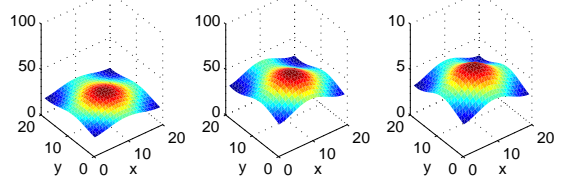

(f) $v_{d}=0.01 \mathrm{x}$ direction

Figure 5: Snapshot of the animation. 